\title{
Comparing the Effects of Standard Suction and Routine Methods on Vital Signs, Arterial Blood Oxygen Saturation and Pain Level of Patients Hospitalized at the Intensive Care Unit
}

\author{
Aliakbar Keykha, ${ }^{1,}$ Hasan Askari, ${ }^{1}$ Abbas Abbaszadeh, ${ }^{2}$ Hasan Enayatie, ${ }^{3}$ Bibi Mahdie Khodadadi \\ Hosini, ${ }^{4}$ and Fariba Borhani ${ }^{2}$ \\ ${ }^{1}$ Community Nursing Research Center, Zahedan University of Medical Sciences, Zahedan, IR Iran \\ ${ }^{2}$ Department of Nursing, Shahid Beheshti University of Medical Sciences, Tehran, IR Iran \\ ${ }^{3}$ Department of Anesthesiology and Intensive Care, Zahedan University of Medical Sciences, Zahedan, IR Iran \\ ${ }^{4}$ Alzahra Eye Hospital, Zahedan University of Medical Sciences, Zahedan, IR Iran \\ "Corresponding author: Aliakbar Keykha, Community Nursing Research Center, Zahedan University of Medical Sciences, Zahedan, IR Iran. E-mail: aiakbar.keykha@gmail.com
}

Received 2016 April 30; Accepted 2016 May 01.

\begin{abstract}
Background: One of the highly important duties at the intensive care units (ICUs) for maintaining airway and improving ventilation and oxygenation is suctioning; however, it must be done in a way so that, in addition to the mentioned advantages, it can have the least complications for the patient.

Objectives: The aim of the present study was to compare the effect of two standard and routine suctioning methods on vital signs, arterial blood oxygen saturation and pain level of patients hospitalized at the ICU.

Materials and Methods: The present quasi-experimental study was conducted during year 2012 on 80 patients under mechanical ventilation hospitalized at ICUs of Zahedan city. Patients were selected purposively and were randomly divided to two groups. In the first group, suctioning was done based on the standard method recommended by the American association for respiratory care (AARC) and in the second group; it was conducted based on routine nursing care. The data was collected by means of Behavioral Pain Scale (BPS) and bedside monitoring of the patients was done before and at one, five, ten and fifteen minutes after suctioning. Results: The results showed that suctioning based on the routine method leads to more pain and this pain continues for 10 minutes after suctioning and there was a statistically significant difference between the two groups at one and five minutes $(\mathrm{P}=0.000, \mathrm{P}=$ 0.000). The vital signs in both groups increased after suctioning and these changes were more in the second group, however, there was no significant difference between the two groups. Also, the level of arterial blood oxygen saturation increased after suctioning in both groups, but these changes were more in group 1 and there was a significant difference between the measurements at one, five, ten and fifteen minutes after suctioning and before suctioning $(\mathrm{P}=0.00, \mathrm{P}=0.000, \mathrm{P}=0.000)$; yet in the second group, no significant difference was observed in none of the times $(\mathrm{P}>0.05)$.

Conclusions: The results of the present study showed that suctioning in the standard method brings about less pain for the patients. It results fewer changes in vital signs and also leads to increased arterial blood oxygen saturation. Thus, it should be included in the work program of the nurses through adequate training.
\end{abstract}

Keywords: Suctioning, Vital Signs, Arterial Blood Oxygen Saturation, Pain

\section{Background}

Respiratory system disorder is the most important challenge at the intensive care unit (ICU); therefore, using mechanical ventilator and tracheal tube is inevitable in these units (1). In adults, $100 \mathrm{~mL}$ of mucus is secreted everyday to wet their airway. Different factors can increase the amount of these secretions through stimulating the airway. Factors that can increase airway secretions include foreign body aspiration, chronic obstructive pulmonary disease, heart surgery and tracheal tube; these factors can increase secretions and cause the inability of secretions discharge (2).
Accumulation of secretions in the respiratory tract causes obstruction of the airways and also causes hypoxia, acidosis and other dangerous complications for the patients through disruption in the oxygen supply (3). Patients with tracheal tube are not able to discharge secretions and they need periodical suctioning (4); tracheal tube suctioning is one of the most important and common methods at the ICU for airway clearance, improvement of oxygenation and prevention of atelectasis and infection (5, 6).

In case of incorrect and non-standard suctioning, complications such as infection, cardiac arrhythmia, reduction of blood oxygen and hypoxemia, increased carbon dioxide, 
damage to the mucous, increased cerebral pressure (ICP) and even cardiac arrest could arise (7). Tracheal suctioning could be done 8 to 17 times routinely in ICU for hospitalized patients (8). To minimize tracheal tube suctioning complications, considering suctioning principles seems necessary. In addition to these principles, choosing an appropriate method for tracheal tube suctioning can be helpful in decreasing its complications (9).

There have been several studies regarding different methods of suctioning. In a study done by Mazhari et al. that compared the effect of open and closed suctioning on physiologic symptoms of patients hospitalized at the ICU, it was reported that changes in heart rate and arterial blood oxygen saturation in the group with open suctioning was more than the group with closed suction. These differences were statistically significant, but suctioning with open or closed methods does not make any change in heart rhythm (9). Also in another study by Lee et al., it was reported that suctioning with the open method causes a greater increase in heart beat immediately after suctioning compared with the closed method, and it also causes a significant decrease of arterial oxygen saturation immediately after suctioning (10).

In another study done by Afshari et al. open and closed suctioning methods were studied, and increase of heart beat was found to be higher in open suctioning compared with closed suctioning; this increase continues $10 \mathrm{~min}$ utes after suctioning, and 15 minutes after suctioning, it returns to its original situation (7). In another study by Vahdatnejad et al. two methods of suctioning with 100 and $200 \mathrm{~mL} \mathrm{Hg}$ pressure were assessed and it was reported that the mean of heart rate, arterial blood pressure and arterial blood oxygen saturation were the same in the two groups (11). Despite several studies regarding comparison of open and closed suctioning methods, there are no studies regarding comparison of the recommended standard method and the routine method used by nurses for tracheal tube suctioning.

\section{Objectives}

Therefore the current study was conducted to compare the effect of standard and routine suctioning methods on vital signs, arterial blood oxygen saturation and pain level of the patients hospitalized at the ICU.

\section{Materials and Methods}

The current study was a semi-experimental study, which was done after obtaining ethical approval from the Kerman Medical Sciences University (code: K.91.138). This study was done on 80 adult patients hospitalized at ICUs of three hospitals in Zahedan during year 2012. Considering patients' lack of consciousness, informed satisfaction was taken from their family members. Inclusion criteria included; being 18 - 65 years old, stable hemodynamic symptoms, lack of receiving inotropic and chronotropic drugs for maintaining pressure and pulse, having a sinus heart rhythm, being hospitalized at least for 48 hours, $\mathrm{fi}_{2} 40 \%$ ventilation in the last 24 hours, positive end-expiratory pressure (PEEP) equal to $5 \mathrm{~cm} \mathrm{H}_{2} \mathrm{O}$ and having no signs of increased ICP. Exclusion criteria included, clear hemodynamic changes, a sharp drop in blood oxygen saturation and need to change $\mathrm{fiO}_{2}$ during the study.

According to the results of the study, 80 samples were required (7). Sampling was done through the purposive method, and samples were divided to two groups with 40 people through random numbers table.

Data collection tools included; a pre-designed form, which contained demographic features, vital signs, arterial blood oxygen saturation and patients' pain score in BPS, which was recorded before suctioning and at 1, 5, 10 and 15 minutes after suctioning in that form. The BPS is an important scale for measuring pain of patients who are hospitalized at ICUs and are not able to have verbal communication. This scale was designed by Dehghani et al. and has three main parts and each part is scored from one to four. The lowest achieved score was three and the highest score was 12 (the highest level of pain) (Table 1). Validity and reliability of these tools were determined by Dehghani et al. on patients with brain injury hospitalized at the ICU (Cronbach's alpha coefficient during painful procedures and non-painful procedures was respectively 0.85 and 0.76); they reported that this tool has appropriate validity and reliability for measuring pain level of brain injury patients who are not able to communicate (12).

All the patients received airway humidification through the same humidifier and same heat. All the patients were suctioned by a central suction aspiration. Indication of suctioning in both groups was increased airway pressure, beginning of ventilator p peak alarm, hearing coarse crackles on tracheal, coughing and observing secretions inside the patient's tracheal tube. In the first group, suctioning was done through the standard method based on the confirmed protocol of American association for respiratory care (AARC), so that before doing tracheal tube suctioning, the patient was hyper-ventilated for one minute with $100 \%$ oxygen. The suctioning procedure was then started. External diameter of the Nelaton catheter was less than half of the internal diameter of the patient's tracheal tube; by considering patient's tracheal tube size and by using sterile gloves, the catheter was brought down to an extent to be less than $2 \mathrm{~cm}$ lower than the patient's 
Table 1. Behavioral Pain Scale (BPS)

\begin{tabular}{|lc|}
\hline Description Item & Score \\
\hline Facial expressions & \\
\hline Calm facial expression & 1 \\
\hline Partially tightened (for example, brow lowering) & 2 \\
\hline Fully tightened (for example, eyelid closing) & 3 \\
\hline Grimacing & 4 \\
\hline Upper limbs & 1 \\
\hline Does not move upper limbs & 2 \\
\hline Limbs are slightly bent & 3 \\
\hline Limbs completely bent with finger flexion & 4 \\
\hline Permanently contractions & 1 \\
\hline Compatibility with ventilator & 2 \\
\hline Tolerating ventilator & 3 \\
\hline Coughing but tolerating ventilator for most of the time & 4 \\
\hline Fighting with ventilator & \\
\hline Unable to tolerating ventilator & \\
\hline
\end{tabular}

${ }^{\mathrm{a}}$ The lowest score: 3 and the highest score: 12.

tracheal tube. The suctioning machine was then switched on and by using the finger tube, suction pressure was controlled from 120 to $150 \mathrm{mmHg}$ and suctioning was done rotationally for 15 to 30 seconds, then Nelaton was removed and the patient was attached to the ventilator (4). Suctioning was done with its usual method in the second group. Latex gloves were used for suctioning in the usual method. Selecting the Nelaton catheter did not follow any specific principles. The green Nelatone or any other available Nelatone catheter that was possible to be entered into the tracheal tube was used.

Patient's hyperventilation with $100 \%$ oxygen was not always done before suctioning. Sometimes Nelaton was entered into the tracheal tube when the suctioning device was off or it was entered into the tracheal tube simultaneously with switching on the suctioning machine, and suctioning was started. Usually an unknown amount of normal saline solution was poured into the tracheal tube through an extension tube attached to a normal saline solution, which was prepared for this purpose. Suctioning was sometimes done without normal saline. Duration of suctioning did not have any specific time and it was stopped when a nurse diagnosed that there was no remaining secretion. Nelaton was inserted into the airway to its end and there was no control on pressure increase. It was observed that the suctioning machine pressure sometimes increased to $400 \mathrm{mmHg}$. Vital signs and arterial blood oxy- gen saturation and BPS score were measured and recorded in both groups before suctioning and at 1, 5, 10 and 15 after suctioning. Data analysis was done through the SPSS 18 software by using independent t-test for comparing intergroup mean scores at different times and Analysis of Variance (ANOVA) of repeated sizes for assessing the effect of time on the achieved scores, and $\mathrm{P}>0.05$ was considered significant.

\section{Results}

Out of 80 patients, there were 64 males and 16 females. Patients' mean age was 32.5 and the standard deviation was 10.6 years. There was no significant statistical difference between the two groups in terms of age and gender through the independent $t$-test $(\mathrm{P}=0.220, \mathrm{P}=0.264)$.

According to the results of the independent t-test, the average pain behavioral score before suctioning was the same in the patients of both groups and there was to statistical significant difference between the two groups $(\mathrm{P}=$ 1). However, there was a significant difference between the two groups in terms of mean BPS at one and five minutes after suctioning. Therefore, the scores average indicated that suctioning increases BPS in patients of group 2 compared with patients of group $1(\mathrm{P}=0.000, \mathrm{P}=0.000)$. The $\mathrm{BPS}$ at 10 and 15 minutes after suctioning was the same in patients of the two groups and there was no significant statistical difference between them $(P=0.32, P=0.186)$.

Assessing time changes was done through ANOVA repeated measures. The BPS mean in group 1 increased after suctioning at all time points (1, 5, 10 and 15 minutes) compared with the initial measurement; this difference was significant only at one and five minutes after suctioning ( $P$ $=0.000, P=0.01)$. The BPS mean of group 2 was the same as group 1 , so that after doing suctioning at all time points $(1,5,10$ and 15 minutes), BPS increased compared with its level before suctioning; this difference was significant at 1 , 5 and 10 minutes after suctioning $(\mathrm{P}=0.000, \mathrm{P}=0.000, \mathrm{P}=$ 0.006).

Independent t-test showed that systolic blood pressure mean before suctioning was the same in both groups and there was no statistical significant difference $(P=0.73)$, also there was no significant difference between the two groups at 1, 5,10 and 15 minutes after suctioning $(\mathrm{P}>0.05)$.

Assessing changes of time in patients of each group through repeated measures ANOVA showed that the mean systolic blood pressure of group 1 at 1, 5 and 10 minutes after suctioning increased compared with the initial measurement, yet this difference was not significant for any of the time points $(\mathrm{P}>0.05)$. Systolic blood pressure decreased 15 minutes after suctioning compared with the initial measurement and this difference was not significant $(\mathrm{P}$ 
$>0.05)$. According to the results of this test, mean systolic blood pressure increased in group 2 after suctioning at all time points $(1,5,10,15$ minutes) compared with its initial value. This difference was significant at 1,5 and 10 minutes after suctioning $(\mathrm{P}=0.000, \mathrm{P}=0.000, \mathrm{P}=0.000)$.

Using independent t-test, there was no significant statistical difference in diastolic blood pressure mean of the two groups before suctioning $(\mathrm{P}=0.80)$. Although diastolic blood pressure mean at all time points after suctioning in group 2 was higher than group 1, this difference was statistically significant only at 15 minutes after suctioning $(\mathrm{P}=0.04)$.

Assessing changes at different time points through repeated measures ANOVA showed that mean diastolic blood pressure in group 1 after suctioning at all time points decreased compared with its initial level, yet this difference was significant only at 15 minutes after suctioning $(\mathrm{P}=$ 0.03). According to the results of this test, mean diastolic blood pressure of group 2 after suctioning increased at all the time points $(1,5,10,15$ minutes $)$ compared with its original value. This difference was significant at 5 and 10 minutes after suctioning $(\mathrm{P}=0.006, \mathrm{P}=0.01)$.

Regarding the mean heart-beat of the two groups before suctioning, there was no statistical significant difference as indicated by the independent t-test $(P=0.34)$. The mean heart rate of group 2 at all time points after suctioning was more than group 1 ; this difference was statistically significant at 1, 5 and 10 minutes after suctioning $(P=0.001$, $\mathrm{P}=0.000, \mathrm{P}=0.002$ ).

Assessing changes at different times was done through repeated measuring ANOVA. The mean heart rate of group 1 after 1 and 5 minutes of suctioning was significantly higher than before suctioning $(\mathrm{P}=0.000, \mathrm{P}=0.009)$. According to the results of this test, the mean heart rate of group 2 after suctioning at all time points (1, 5, 10 and 15 minutes) were significantly higher than its original measurement $(\mathrm{P}$ $=0.000, \mathrm{P}=0.000, \mathrm{P}=0.000, \mathrm{P}=0.004)$.

The mean $\mathrm{O}_{2}$ saturation before suctioning in patients of the two groups, as indicated by the independent t-test, was not statistically significant $(\mathrm{P}=0.24)$. The mean $\mathrm{O}_{2}$ sat of the patients in group 1 at all time points was more than patients in group 2, yet this difference was statistically significant only at 5 minutes after suctioning $(\mathrm{P}=0.04)$.

Assessing changes at different times in patients of each group was done through repeated measuring ANOVA. The average $\mathrm{O}_{2}$ saturation of group 1, after suctioning at 1, 5, 10 and 15 minutes was significantly higher than its initial measurement $(\mathrm{P}=0.001, \mathrm{P}=0.000, \mathrm{P}=0.000)$. According to the results of this test, the mean $\mathrm{O}_{2}$ sat of group 2 at 10 and 15 minutes after suctioning increased slightly, but this increase, compared with before suctioning, was not statistically significant $(\mathrm{P}>0.05)$ (Figures 1 and 2 ).

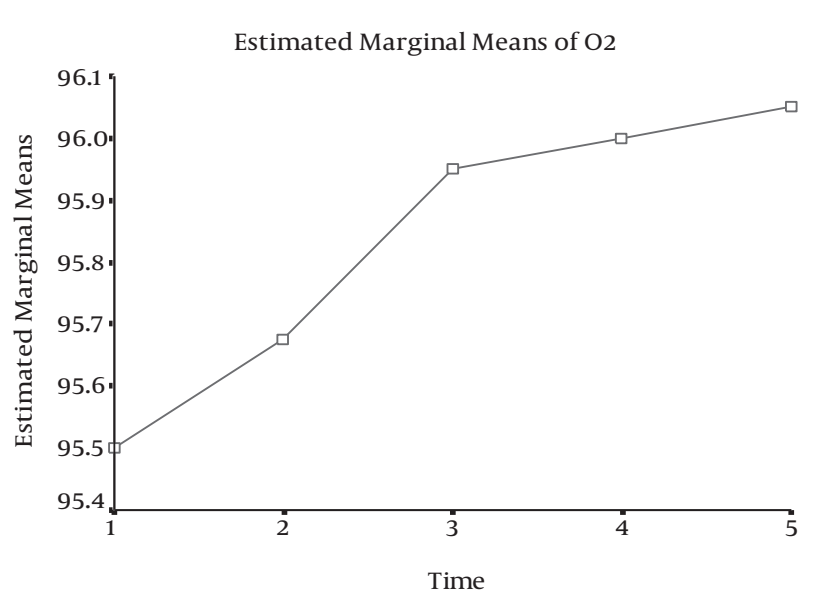

Figure 1. Changes in Arterial Blood Oxygen Saturation by Standard Suctioning Group

\section{Discussion}

The results of the current study showed that suctioning increases pain and agitation in patients and this increase in group two was more than group 1 for up to $10 \mathrm{~min}$ utes, which is consistent with the study of Akbar Keykha et al. their study compared two methods of relieving pain. Increase of agitation and pain were observed in both groups after suctioning (13). In their study, patients of both groups received the same method of suctioning, while in this study, different suctioning methods were used, however suctioning increased pain and agitation; several factors such as depth, time and pressure of suctioning can be among important and effective factors of pain and agitation, considering the issue that these factors were more controlled in the first group, level of pain and agitation in the first group was less than the second group. Also in the study of Sole et al. it was shown that suctioning increases patients' pain and agitation (14). Furthermore, the results of the study of Rahu et al. showed that suctioning increases pain and agitation and there was a significant difference between the measurements before suctioning and after suctioning in this regard (15).

Suctioning also caused some changes regarding vital signs; systolic blood pressure had a little increase after standard suctioning at all times in group 2, but this difference was not statistically significant; however, this increase was higher in group 2 and there was a significant difference compared with the measurement before suctioning at all times. Regarding diastolic pressure in group 1, decreased diastolic pressure was observed at all time points after suctioning compared with its initial value, while in group 1, in which the usual suctioning of the ward was 


\section{Estimated Marginal Means of $\mathrm{O} 2$}

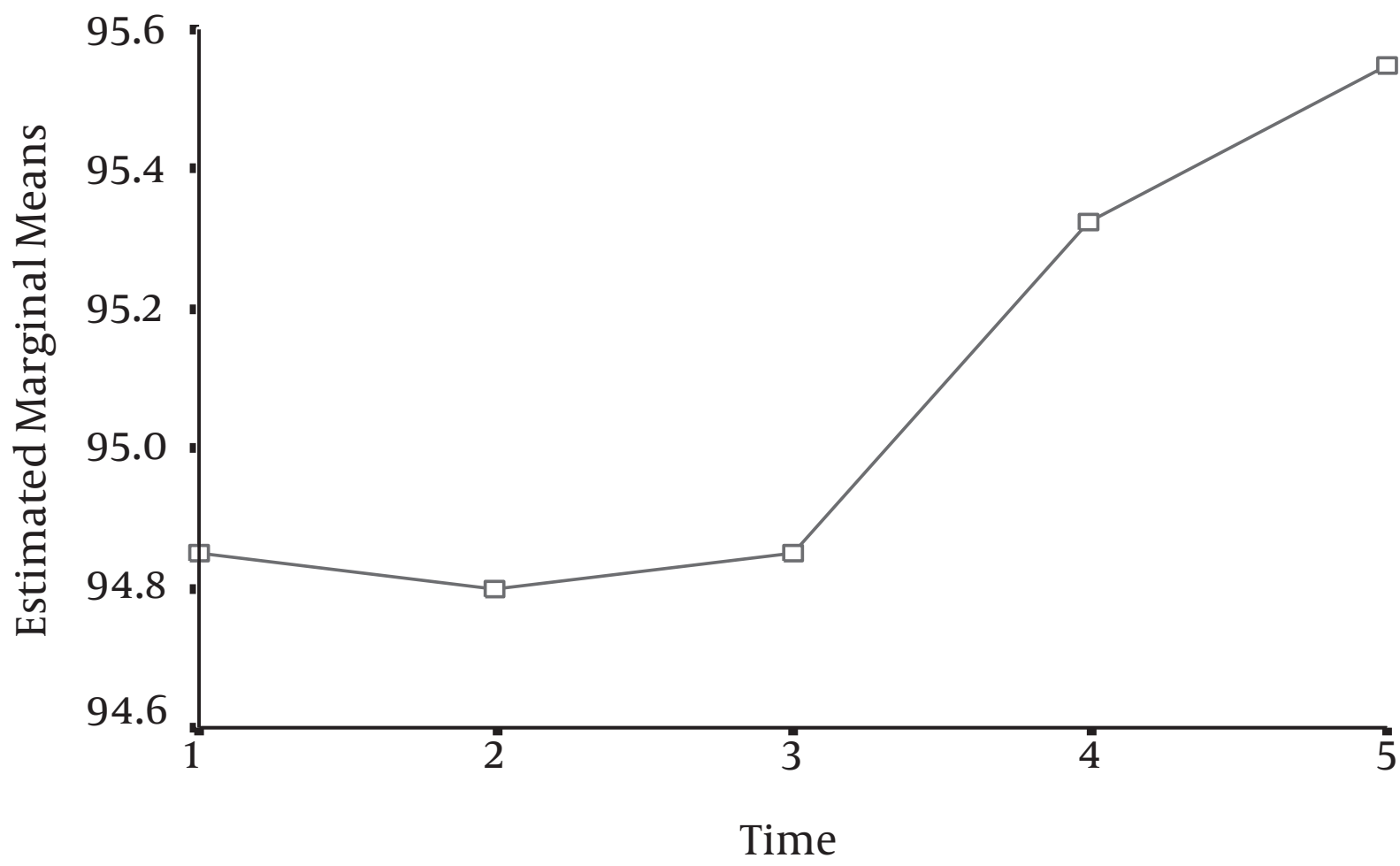

Figure 2. Changes of Arterial Blood Oxygen Saturation Amount in the Routine Suctioning Group

done, we observed increased diastolic blood pressure at all time points compared with before suctioning. In the study of Abbas-zadeh et al. in both groups, systolic and diastolic pressure was increased after suctioning, which is not completely consistent with the results of this study. We observed decreased diastolic pressure in one group, although the type of suction method in this study was different in this group (13).

In the study of Nazmiyeh et al. which compared two methods of closed and open suctioning, increased systolic pressure was observed. This decrease continued for ten minutes in both groups, and then the process was reversed. We also observed increased diastolic pressure only at one minute after suctioning, while at other times, we observed decreased diastolic pressure compared with before suctioning in both groups; this decrease was higher with open suctioning. We also observed decreased diastolic pressure in group 1 , which is consistent with the mentioned study, although in our study, both groups were suctioned through the open suctioning method (16).

Suctioning also caused increased heart rate in both groups, but in group 1 heart rate returned to the base sta- tus after five minutes, while this increase of heart rate continued for 15 minutes and was not close to the base level and the difference was significant at all stages compared with before suctioning. This difference can be related to depth, time or pressure of suctioning and also use of normal saline in group 2. In the study of Iranmanesh et al. increased heart rate occurred in both groups that received suctioning with or without normal saline and this increase was more in the group that used normal saline, which is consistent with the results of this study (17).

Arterial blood oxygen saturation of the patients increased after suctioning in both groups. This increase occurred with more delay in group 1 and was started 10 minutes after suctioning and there was no significant difference compared with the value before suctioning; this is while, there was a significant difference in group 1 at all times. In the study of Sole et al. in which suctioning of the tracheal tube was done with the standard method, increased arterial blood oxygen saturation occurred after suctioning, which is consistent with the results of this study (14).

In the study of Iranmanesh et al. negative changes 
in $\mathrm{O}_{2}$ saturation was observed in the group that received suctioning with normal saline. In this study, decreased arterial blood oxygen saturation was not observed, but increased oxygen saturation occurred with greater delay in group 2 (17). In the study of Akbaryan Deheki et al. which compared three methods of suctioning with normal saline, N-acetyl cysteine and without them, it was reported that arterial blood oxygen saturation decreased at 2 and 5 minutes after suctioning in all the three groups; this was while the level of heart rate and blood pressure decreased at these times compared with before suctioning, which is not consistent with the results of the present study. We did not observe decreased arterial blood oxygen saturation in none of the groups, there was only a slight delayed increase in the routine suctioning group; we also observed increased pulse and blood pressure after suctioning compared with the initial values (2).

\subsection{Conclusions}

Suctioning with the standard method results in a slight change in vital signs, and causes less pain and agitation. Also using this method causes increased arterial blood oxygen saturation sooner than the routine method. Therefore using this method through adequate education should be included in the nurses' schedule. Considering complications due to the usual method and short duration of this study, it is recommended to conduct a long-term study to assess the relationship between type of suctioning and pneumonia due to the ventilator.

\section{Acknowledgments}

The researchers thank and appreciate the vice counselor of research of Medical Sciences University of Kerman and Zahedan and treatment management of the Social security organization of Zahedan and the staff of the ICU.

\section{References}

1. Lorente L, Lecuona M, Martin MM, Garcia C, Mora ML, Sierra A. Ventilator-associated pneumonia using a closed versus an open tracheal suction system. Crit Care Med. 2005;33(1):115-9. [PubMed: 15644657].

2. Akbaryan Deheki N, Sanagoo A, Amri P, Moghaddam S, Vakili MA, Nasiri $\mathrm{H}$, et al. Comparing the effect of using normal saline, $\mathrm{N}$-acetyl cysteine and not using them in endotracheal tube suction on physiologic parameters and the amount of secretions in intubated patients under mechanical ventilation. Iran J Crit Care Nurs. 2014;6(4):152-9.
3. Burns SM, Chulay M. AACN Essentials of Critical Care Nursing McGraw-Hill Medical; 2010.

4. Inan D, Saba R, Yalcin AN, Yilmaz M, Ongut G, Ramazanoglu A, et al. Device-associated nosocomial infection rates in Turkish medical-surgical intensive care units. Infect Control Hosp Epidemiol. 2006;27(4):343-8. doi: 10.1086/503344. [PubMed:16622810].

5. Subirana M, Sola I, Benito S. Closed tracheal suction systems versus open tracheal suction systems for mechanically ventilated adult patients. Cochrane Database Syst Rev. 2007(4):CD004581. doi: 10.1002/14651858.CD004581.pub2. [PubMed: 17943823].

6. Etemadifar SH, Nemati SH, Aslani Y, Mehr A. Effects of Intratracheal Suctioning on Hemodynamic Parameters and Arterial Oxygen. Iran J Nurs. 2008;21(54).

7. Soltanian A. Comparison the effect of open and closed endotracheal suctioning systems on heart disrhythmia in mechanically ventilated patients. Sci J Hamadan Nurs Midwifery Faculty. 2014;21(4):5-11.

8. Jongerden IP, Rovers MM, Grypdonck MH, Bonten MJ. Open and closed endotracheal suction systems in mechanically ventilated intensive care patients: a meta-analysis. Crit Care Med. 2007;35(1):26070. doi: 10.1097/01.CCM.0000251126.45980.E8. [PubMed: 17133187].

9. Mazhari S, Pishgou'ei AH, Zareian A, Habibi H. Effect of open and closed endotracheal suction systems on heart rhythm and artery blood oxygen level in intensive care patients. J Crit Care Nurs. 2010;2(4):1-2.

10. Lee CK, Ng KS, Tan SG, Ang R. Effect of different endotracheal suctioning systems on cardiorespiratory parameters of ventilated patients. Ann Acad Med Singapore. 2001;30(3):239-44. [PubMed: 11455735].

11. Vahdatnejad J, Abbasinia M, Hoseinpoor SH, Babaii A. The comparison between two methods of endotracheal tube suctioning with negative pressure of 100 and 200 mmhg. Iran J Nurs Res. 2014;9(3):68-75.

12. Dehghani H, Tavangar H, Ghandehari A. Validity and reliability of behavioral pain scale in patients with low level of consciousness due to head trauma hospitalized in intensive care unit. Arch Trauma Res. 2014;3(1):e18608. doi: 10.5812/atr.18608. [PubMed: 25032173].

13. Akbar Keykha A, Abbaszadeh A, Enayati H, Borhani F, Rafiei H, Khodadadi Hoseini BM. Applying the instruction of pain control and sedation of the patients hospitalized in intensive care unit. Iran J Crit Care Nurs. 2014;6(4):243-50.

14. Sole ML, Bennett M, Ashworth S. Clinical Indicators for Endotracheal Suctioning in Adult Patients Receiving Mechanical Ventilation. Am J Crit Care. 2015;24(4):318-24. doi: 10.4037/ajcc2015794. [PubMed: 26134331] quiz 325.

15. Rahu MA, Grap MJ, Cohn JF, Munro CL, Lyon DE, Sessler CN. Facial expression as an indicator of pain in critically ill intubated adults during endotracheal suctioning. Am J Crit Care. 2013;22(5):412-22. doi: 10.4037/ajcc2013705. [PubMed: 23996421].

16. Nazmiyeh H, MirJalili MR, Emami Maibodi R. Comparison of the ef fects of open and closed endotracheal suction on cardiovascular and ventilation parameters for patients undergoing Mechanical ventilation.J Rafsanjan Univ Med Sci. 2010;9(2):97-106.

17. Iranmanesh $\mathrm{S}$. Comparison of the endotracheal tube suctioning with and without normal saline solution on heart rate and oxygen saturation. Iran J Crit Care Nurs. 2011;4(3):117-20. 